\title{
Higher macrophage superoxide anion production in coronary artery disease (CAD) patients with Type D personality
}

Short title: Type D, CAD, and macrophage activity

${ }^{\mathrm{a}, \mathrm{b}}$ Claudia Zuccarella-Hackl, M.S., ${ }^{\mathrm{d}, \mathrm{c}, \mathrm{b}}$ Roland von Känel, M.D., ${ }^{\mathrm{e}, \mathrm{a}}$ Livia Thomas, M.S., ${ }^{\text {a }}$ Peggy Kuebler, B.S., ${ }^{\mathrm{f}}$ Jean-Paul Schmid, M.D., ${ }^{\mathrm{c}}$ Heinrich P. Mattle, M.D., ${ }^{\mathrm{c}}$ Marie-Louise Mono, M.D., ${ }^{b}$ Robert Rieben, Ph.D., ${ }^{\mathrm{g}}$ Roland Wiest, M.D., \& ${ }^{\mathrm{e}, \mathrm{a}, \mathrm{b}}$ Petra H. Wirtz, Ph.D.

${ }^{a}$ Biological and Health Psychology, University of Bern, Bern Switzerland

${ }^{b}$ Department of Clinical Research, University of Bern, Bern Switzerland

${ }^{c}$ Department of Neurology, Inselspital, Bern University Hospital, and University of Bern, Bern Switzerland

${ }^{d}$ Department of Psychosomatic Medicine, Clinic Barmelweid, Barmelweid, Switzerland

${ }^{e}$ Biological Work and Health Psychology, University of Konstanz, Konstanz, Germany

${ }^{f}$ Cardiology Clinic, Tiefenauspital, Bern University Hospital, Bern, Switzerland

${ }^{g}$ Institute of Diagnostic and Interventional Neuroradiology, University Hospital Bern, and University of Bern, Bern Switzerland

Total word count: 5739

Address for correspondence and reprint requests:

Petra H. Wirtz, Ph.D., Work and Health Psychology, University of Konstanz, Universitaetsstrasse 10, 78457 Konstanz, Germany, Phone: +49 7531 88-3742; Fax: +49 7531 88-5028; Email address: petra.wirtz@uni-konstanz.de 


\section{SUMMARY}

Background: Type D personality (Type D) is an independent psychosocial risk factor for poor cardiac prognosis and increased mortality in patients with cardiovascular disease (CVD), but the involved mechanisms are poorly understood. Macrophages play a pivotal role in atherosclerosis, the process underlying CVD. We investigated macrophage superoxide anion production in coronary artery disease $(\mathrm{CAD})$ patients with and without Type D.

Methods and Results: We studied 20 male CAD patients with Type D (M:66.7 $\pm 9.9 y e a r s)$ and 20 agematched male CAD patients without Type D (M:67.7 $\pm 8.5 y e a r s)$. Type D was measured using the DS14 questionnaire with the two subscales 'negative affectivity' and 'social inhibition'. We assessed macrophage superoxide anion production using the WST-1 assay. All analyses were controlled for potential confounders. CAD patients with Type D showed higher superoxide anion production compared to CAD patients without Type D $(\mathrm{F}(1,38)=15.57, p<.001)$. Complementary analyses using the Type D subscales 'negative affectivity' and 'social inhibition', and their interaction as continuous measures, showed that both Type D subscales (negative affectivity: $\left(\beta=.48, p=.002, R^{2}=.227\right.$ ); social inhibition: $\left(\beta .46, p=.003, R^{2}=.208\right)$ and their interaction $\left(\beta=.36, p=.022, R^{2}=.130\right)$ were associated with higher WST-1 reduction scores. Results remained significant when controlling for classical CVD risk factors (i.e. body mass index, mean arterial blood pressure), atherosclerosis severity (i.e. intima media thickness, presence of carotid plaques), and psychological factors (depressive symptom severity, chronic stress).

Conclusions: Our results indicate higher macrophage superoxide anion production in CAD patients with Type D compared to those without Type D. This may suggest a mechanism contributing to increased morbidity and mortality in CAD patients with Type D.

Key words: human macrophages, NADPH oxidase, superoxide anions, CAD, Type D personality. 


\section{INTRODUCTION}

Inflammatory processes play a pivotal role in the progression of coronary artery disease (CAD) and its underlying process atherosclerosis (Libby, 2002). Macrophages are tissue-resident phagocytic immune cells derived from circulating blood monocytes (Lucas \& Greaves, 2001). A key initial event of coronary atherosclerosis is the entry of monocytes into the arterial intima, where they mature into macrophages. Intimal macrophages phagocyte oxidized lipoproteins and eventually differentiate into foam cells, a critical and prevalent component of atherosclerotic plaques. Moreover, macrophages are important mediators of inflammation in atherosclerosis (Moore et al., 2013; Robbins et al., 2013).

Microbicidal activity, i.e. the killing of microbes, is a key innate immune effector function of classically activated macrophages also known as inflammatory M1 macrophages (Martinez et al., 2008; Mosser \& Edwards, 2008). Macrophage microbicidal activity is largely mediated by increased secretion of microbe killing oxidizing agents termed reactive oxygen species (ROS; De OliveiraJunior et al., 2011; Halliwell, 2006). ROS production in turn derives from activation of the multisubunit enzyme nicotinamide adenine dinucleotide phosphate (NADPH) oxidase located in the phagolysosome and plasma membrane of macrophages. Once activated, NADPH oxidase transfers electrons from NADPH in the cytosol to extracellular or intraphagolysosomal oxygen molecules. These oxygen molecules are then chemically reduced to highly reactive superoxide anions and other ROS subtypes (De Oliveira-Junior et al., 2011; Cathcart, 2004). In particular, superoxide anions are of major importance for the microbicidal activity of macrophages in host defense (Mosser \& Edwards, 2008; Nathan \& Shiloh, 2000).

Increasing evidence suggests that NADPH oxidase and the resulting production of superoxide anions are likely to play a critical role in the pathogenesis of atherosclerosis. For instance, NADPH oxidase-derived superoxide anions can induce low-density lipoprotein oxidation (Cathcart, 2004; Cai \& Harrison, 2000; Griendling et al., 2000) an important cause of endothelial dysfunction as an initial step in atherosclerosis (Libby, 2002; Libby et al., 2011). Indeed, NADPH oxidase-deficient mice developed significantly smaller atherosclerotic lesions (Barry-Lane et al., 2001). Compared with healthy controls, CAD patients had greater NADPH oxidase activation and subsequent superoxide 
anion production in coronary arteries. This ROS increase was partly related to higher monocyte/macrophage infiltration (Guzik et al., 2006). Moreover, in CAD patients, superoxide anion production by stimulated neutrophils was enhanced with increasing arterial stiffness, a marker of adverse cardiovascular prognosis (Wykretowicz et al., 2005).

Different psychosocial factors significantly relate to the pathogenesis of CAD (Chida \& Steptoe, 2009; Rosanski, 2014) and have been identified as risk factors for cardiac events both in healthy subjects and CAD patients (Chida \& Steptoe, 2009; Lichtman et al., 2008). Type D ("distressed") personality (Type D) is defined as a tendency to experience negative emotions, and to inhibit their expression in a social context (Denollet et al., 1996). Evidence suggests that Type D is an independent predictor of cardiovascular morbidity and mortality in patients with CAD (Denollet et al., 2013; Grande et al., 2004; O’Dell et al., 2011). However, potential mechanisms underlying poor cardiac prognosis with Type D are unclear and may include inflammatory processes (Denollet et al., 2003; Kupper \& Denollet, 2007). Indeed, two studies with chronic heart failure (CHF) patients found Type D to independently predict increased circulating levels of tumor necrosis factor (TNF)- $\alpha$ and TNF- $\alpha$ soluble receptors (Conraads et al., 2006; Denollet et al., 2003). Of note, TNF- $\alpha$ is a proinflammatory cytokine involved in atherosclerosis development and progression (Libby, 2002) and also in the pathogenesis of CHF (Aukrust et al., 2005). Furthermore, in CHF patients Type D was associated with increased oxidative stress evidenced by assessing heat shock protein (HSP) 70 and xanthine oxidase (Kupper et al., 2009).

As yet, superoxide anion production in CAD patients with Type D has not been studied. Therefore, the aim of this study was to compare phagocytic NADPH oxidase-derived superoxide anion production by inflammatory M1 macrophages between CAD patients with and without Type D. To rule out potential confounding effects of age on superoxide anion production (Fulop et al., 2004, Shaw et al., 2013), we recruited CAD patients with and without Type D individually matched on age. Given the important role of both superoxide anion production and Type D in the pathogenesis and progression of $\mathrm{CAD}$, we hypothesized that Type $\mathrm{D}$ patients would show higher superoxide anion production when compared with non-Type D patients. 


\section{MATERIALS AND METHODS}

\subsection{Patients with coronary artery disease}

This study was part of a larger project assessing psychobiological mechanisms in patients with CAD. We contacted male patients who were diagnosed with CAD at the Cardiac Prevention and Rehabilitation Clinic, Bern University Hospital, Switzerland, at least 6 months previously. Patients interested to participate were screened by telephone interview using an extensive health questionnaire. Explicit exclusion criteria were: regular strenuous exercise, alcohol and illicit drug abuse, liver and renal diseases, chronic obstructive pulmonary disease, allergies and atopic diathesis, rheumatic diseases, HIV, cancer, psychiatric and neurological diseases, and current infectious diseases. Between December 2012 and June 2015, we enrolled a total of 101 male patients with a diagnosis of CAD who were also assessed for Type D (see below). For every patient classified as Type D, we recruited an age-matched CAD patient without Type D. Given well-known effects of age on various parameters of the immune system (e.g. Fulop et al., 2004; Shaw et al., 2013), we decided for an age-matched design allowing us to minimize potential confounding of macrophage superoxide anion production by age. In total, we identified 20 eligible CAD patients with Type D personality and recruited 20 eligible age-matched CAD patients without Type D. For one 38-year-old CAD patient with Type $\mathrm{D}$, we were unable to recruit an age-matched CAD patient without Type D; instead, we recruited an older control patient. Data of CAD patients were extracted from hospital charts and included the diagnosis of myocardial infarction (MI), left ventricular ejection fraction (LVEF), coronary artery bypass graft surgery $(\mathrm{CABG})$, and the number of diseased coronary vessels (i.e., stenosis of at least 50\%). Missing data regarding the number of diseased vessels from 4 patients were estimated using the expectation-maximization (EM) algorithm (Moon, 1996). The project was approved by the Ethics Committee of the Canton of Bern, Switzerland and the study protocol is in accordance with the Declaration of Helsinki. All study procedures were carried out with adequate understanding and written informed consent of all participants.

\subsection{Procedure}


Eligible CAD patients were asked to abstain from caffeine and alcohol consumption for $24 \mathrm{~h}$ before testing. They were briefed to consume a breakfast following standardized instructions prior to arrival at our lab at $0800 \mathrm{~h}$. After patients had completed the Type D and other questionnaires, they provided blood samples obtained by short-term cannula insertion (see Kuebler et al., 2013) for the assessment of macrophage superoxide anion production at about 11:30h, (i.e., after a fasting period of $3.5 \mathrm{~h}$ since arrival). In addition, participants underwent duplex ultrasonography for the assessment of intima media thickness (IMT) of the carotid common artery (CCA) and presence of carotid plaques as a measure of atherosclerotic disease severity. Moreover, blood pressure (BP) was assessed by means of sphygmomanometry (Omron M6; Omron Healthcare Europe B.V., Hoofdorp, Netherlands) 3h and $2.5 \mathrm{~h}$ before and $10 \mathrm{~min}$ after the blood samples were obtained. Due to technical problems, one CAD patient with Type D provided only two instead of three BP measurements.

\subsection{Psychological Assessment}

\subsubsection{Type D Personality}

Type D was assessed using the validated German version of the 14-item Type D Scale (DS14; Grande et al., 2004). The DS14 includes the two Type D subscales negative affectivity (NA, e.g. "I am often in a bad mood") and social inhibition (SI, e.g. "I often feel inhibited in social interactions") consisting of seven items each. Items are rated on a 5-point Likert scale ( $0=$ "false", 1="rather false", $2=$ "neutral", $3=$ "rather true", 4="true"). The total scores for each subscale range between 0 and 28 . Type D is classified if both subscales are greater or equal to 10 (Denollet, 2005). Cronbach's alpha of the subscales was .88 for NA and .86 for SI (Denollet, 2005) and .89 for NA and .81 for SI in our sample. In addition, the interaction of continuous NA and SI $Z$ scores was assessed as continuous single measure of Type D (Denollet et al., 2013).

\subsubsection{Depressive Symptom Severity}

Depressive symptom severity was measured using the validated German version (Hautzinger et al., 2006) of the 21-item Beck Depression Inventory - Second Edition (BDI-II; Beck et al., 1996). The BDI was developed for the assessment of depressive symptoms that correspond to the Diagnostic and 
Statistical Manual of Mental Disorders, Fourth Edition criteria for major depressive disorders and measures a somatic and a cognitive-affective dimension of depression (Beck et al., 1996). The BDI-II assesses the frequency and/or severity of symptoms related to sadness, feelings of guilt, perceptions of self-worth, suicidal ideation, and changes in appetite and body weight, among other characteristics. Items are rated on a 4-point Likert scale ranging from 0 (symptom not present) to 3 (symptom very present) that add to a total BDI-II score ranging from 0 to 63 . Higher scores mean higher depressive symptom severity. Cronbach's alpha of the BDI-II total score was between .84 and .91 for nonpsychiatric populations (Hautzinger et al., 2006) and .86 in our sample.

\subsubsection{Chronic Stress}

To assess chronic stress we used the 12-item Chronic Stress Screening Scale (CSSS; Schulz et al., 2004). The CSSS includes questions about frequency of experiencing work overload (four items), worries (four items), lack of social recognition (two items), excessive demands at work (1 item) and social overload (1 item). Items have a 5-point rating format reflecting frequency (1="never" to 5="very often"). Possible scores range from 12 to 60 with higher scores indicating greater chronic stress. Cronbach's alpha of the CSSS total score was .87 (Schulz et al., 2004) and .94 in our sample. Missing CSSS scores of 3 patients with Type D were estimated using the expectation-maximization (EM) algorithm (Moon, 1996).

\subsection{Macrophage activation assessment}

\subsubsection{Reagents and Chemicals}

We used the following reagents: Ficoll-Paque PLUS (Ficoll; no. 17-1440-02; GE Healthcare, Uppsala, Sweden), 2-(4-iodophenyl)-3-(4-nitrophenyl)-5-(2,4-disulfophenyl)2H-tetrazolium (WST-1; no. 150849-52-8; Dojindo Laboratories, Kumamoto, Japan), interferon- $\gamma$ (IFN- $\gamma$; no. PHC4031; Invitrogen, Basel, Switzerland), TNF- $\alpha$ (no. PHC3016; Invitrogen, Basel, Switzerland), Hank's balanced salt solution without phenol red (HBSS; no. 14025050; Invitrogen, Basel, Switzerland), fetal bovine serum (FBS; no. 10270-106; Invitrogen, Basel, Switzerland), lipopolysaccharide (LPS; no. L6529; Sigma-Aldrich, Buchs, Switzerland), phosphate-buffered saline (PBS; no. P5368; Sigma- 
Aldrich, Buchs, Switzerland), phorbol 12-myristate 13-acetate (PMA; no. P8139; Sigma-Aldrich, Buchs, Switzerland), and RPMI 1640 medium with glutamax (RPMI 1640; no. W9925E; Fisher Scientific, Wohlen, Switzerland).

\subsubsection{WST-1 assay}

We assessed the microbicidal potential of ex vivo isolated human monocyte-derived M1 macrophages (HMDM) based on our recent assay validation procedure (Kuebler et al., 2013). In brief, 9ml of blood were collected in EDTA-coated tubes (Sarstedt, Numbrecht, Germany), immediately layered on top of $10 \mathrm{ml}$ Ficoll, and centrifuged for $20 \mathrm{~min}$ at $300 \mathrm{~g}$ and $20^{\circ} \mathrm{C}$. After centrifugation, PBMCs were removed from the interface, washed twice in warm RPMI-1640 medium, counted with a hematologic analyzer (KX-21N; Sysmex Digitana AG), and re-suspended to a final concentration of 3.0x10\% $/ \mathrm{ml}$ with RPMI1640 media supplemented with 10\% FBS. Then, PBMC suspension aliquots of $1 \mathrm{ml}$ were transferred to 24-well cell culture plates (no. 4609; Semadeni; Ostermundigen, Switzerland). After incubation for 2 hours at $37^{\circ} \mathrm{C}$ and $5 \% \mathrm{CO}_{2}$, the supernatant was discarded and the plate surface was rinsed five times with $1 \mathrm{ml}$ of warm $\left(25^{\circ} \mathrm{C}\right) 0.01 \mathrm{M}$ PBS to remove non-adherent PBMCs, while monocytes remained adherent to the bottom of the plate.

The adherent monocyte layer was then diluted with $1 \mathrm{ml}$ RPMI1640 media supplemented with 10\% FBS. Subsequently, we added IFN- $\gamma$, TNF- $\alpha$, and LPS in a final concentration of $20 \mathrm{ng} / \mathrm{ml}$ IFN- $\gamma$, 20ng/ml TNF- $\alpha$, and 300ng/ml LPS to promote differentiation of monocytes into inflammatory macrophages. After incubation for $44 \mathrm{~h}$ at $37^{\circ} \mathrm{C}$ and $5 \% \mathrm{CO}_{2}$, the supernatant was discarded and the adherent macrophage layer was washed twice with $1 \mathrm{ml}$ of warm $\left(25^{\circ} \mathrm{C}\right) 0.01 \mathrm{M}$ PBS to remove traces of culture media and non adherent cells.

Next, the resulting macrophage monolayer (obtained as described above) was overlaid with $1 \mathrm{ml}$ HBSS. Subsequently, IFN- $\gamma$, TNF- $\alpha$, LPS, WST-1 and PMA were added, resulting in a final concentration of $20 \mathrm{ng} / \mathrm{ml}$ IFN- $\gamma, 20 \mathrm{ng} / \mathrm{ml}$ TNF- $\alpha, 300 \mathrm{ng} / \mathrm{ml}$ LPS, 100M WST-1 and 50nM PMA. Following an incubation period of 4 hours at $37^{\circ} \mathrm{C}$ and $5 \% \mathrm{CO}_{2}$, the supernatant was removed and used to determine WST-1 reduction by reading the optical densities (ODs) with a spectrophotometer (Tecan infinite M1000, Tecan, Salzburg, Austria) at $450 \mathrm{~nm}$ against water as a blank. Higher ODs, as 
obtained in absorbance reading, are associated with higher amounts of WST-1 reduction and thus of superoxide anions generated by HMDM. In 2 CAD patients with Type D and 8 CAD patients without Type D the assays were not performed in duplicates due to low PBMC numbers.

\subsection{Carotid ultrasound}

Participants underwent carotid ultrasound at the Department of Neurology at the Bern University Hospital to assess CCA IMT (mm) and presence of carotid plaques (yes/no) (Naqvi \& Lee, 2014). A Toshiba Aplio 500 scanner (Toshiba Medical Systems, Nasu, Japan), equipped with a 12-MHz linear array transducer, was used by trained sonographers to image carotid arteries. High-resolution B-mode ultrasound images were collected from the far walls of the right and left CCA, and carotid bifurcation, whereby B-mode measurements were averaged over at least $1 \mathrm{~cm}$ segment. Presence of carotid plaques was defined as focal region with carotid artery IMT greater than $1.5 \mathrm{~mm}$ that protrudes into the lumen distinct from the adjacent boundary.

In our analyses we controlled for the mean maximum IMT from far walls of right and left CCAs (mean max IMT) and for presence of carotid plaques.

\subsection{Statistical analysis}

Data were analyzed using SPSS (Version 20) statistical software package for Macintosh (IBM SPSS Statistics, NY, USA). All analyses were two-tailed, with level of significance at $p<.05$. Results are shown as mean \pm SEM.

Prior to statistical analyses, linear data were tested for normal distribution and homogeneity of variance using Kolmogorov-Smirnov and Levene's tests in both groups. All data were normally distributed. For group comparisons WST-1 reduction scores had to be logarithmically transformed to verify homogeneity of variance. For reasons of clarity, we show original WST-1 reduction scores in all figures. We calculated mean arterial blood pressure (MAP) by the formula (2/3 mean diastolic $\mathrm{BP})+(1 / 3$ mean systolic $\mathrm{BP})$. Body mass index (BMI) was calculated as the ratio of weight in kilograms to height in square meters. 
We used analysis of variance (ANOVA) to test for differences in the characteristics of the two groups and to test whether CAD patients with Type D showed higher WST-1 reduction scores as compared to CAD patients without Type D. Complementary analyses comprised multivariate linear regression analyses (enter method) with continuous measures of the Type D subscales and their interaction and tested whether WST-1 reduction scores (dependent variable) were associated with NA, SI, or their interaction, respectively (independent variable). In all analyses with WST-1 reduction scores, we controlled in our age-matched participants for the classical CVD risk factors BMI and MAP, for the atherosclerosis severity parameters IMT and presence of carotid plaques. We additionally covaried for BDI and CSSS, because we previously found both these psychological variables to be related to macrophage superoxide anion production (Kuebler et al., 2013). We restricted the number of control variables of the main analyses to four in order to avoid overcontrolling given our sample size (Babyak, 2004). We selected these control variables a priori based on previous literature on their associations with immune activation or microbicidal potential of HMDM, respectively (Dorshkind et al., 2009; Kuebler et al., 2013; Watanabe et al., 2006; Wirtz et al., 2008; Wirtz et al., 2004).

\section{RESULTS}

\subsection{Group characteristics}

Table 1 shows demographic, medical, and psychological characteristics of the 40 participants studied. The two study groups did not significantly differ in terms of age, BMI, MAP, IMT, or presence of carotid plaques. CAD patients with Type D had higher BDI and CSSS scores than those without Type D.

\subsection{Type D groups and WST-1 reduction scores}

Figure 1 shows that CAD patients with Type D had higher WST-1 reduction scores compared to CAD patients without Type $\mathrm{D}$, either without $\left(\mathrm{F}(1,38)=15.57, p<.001, \mathrm{Eta}^{2}=0.30, f=0.65\right)$ or with controlling for $\mathrm{BMI}$ and MAP $\left(\mathrm{F}(3,36)=14.45, p=.001, \mathrm{Eta}_{\mathrm{p}}{ }^{2}=0.29, f=0.64\right)$. Additional controlling for IMT or presence of carotid plaques $(p ' s=.001)$ did not significantly change results. 
Controlling for BDI, CSSS, or both BDI and CSSS, in addition to BMI and MAP did also not change results ( $p$ 's $\leq .009$ ), even if IMT or presence of carotid plaques were also controlled ( $p$ 's $\leq .010)$.

\subsection{Associations between WST-1 reduction scores and Type D subscales}

Complementary analyses using the Type D subscales NA and SI, or their interaction as continuous measures showed that higher scores on both Type D subscales and their interaction were associated with greater WST-1 reduction scores without and with controlling for potential confounders.

\subsubsection{Negative affectivity}

In detail, higher NA was significantly associated with higher WST-1 reduction scores $(\beta=.48, p$ $=.002, R^{2}=.227$; see Figure $2 \mathrm{~A}$ ). This effect remained significant after controlling for BMI and MAP $\left(\beta=.48, p=.002, \Delta R^{2}=.229\right)$. Also, additional controlling for IMT or plaque occurrence ( $p$ 's $\leq .003)$ did not significantly change these results. Results remained significant after controlling for BDI, CSSS, or both BDI and CSSS, in addition to BMI and MAP ( $p$ 's $\leq .012)$, even if IMT or presence of carotid plaques were also controlled ( $p$ 's $\leq .013)$.

\subsubsection{Social inhibition}

Similar results were obtained for SI: Higher SI significantly related to higher WST-1 reduction scores $\left(\beta=.46, p=.003, R^{2}=.208\right.$; see Figure 2B). This effect was independent of BMI and MAP $(\beta=.45, p$ $\left.=.005, \Delta R^{2}=.197\right)$, even while also controlling for IMT or presence of carotid plaques ( $p$ 's $\left.=.005\right)$. Again, these results held significance after controlling for BDI, CSSS, or both BDI and CSSS in addition to BMI and MAP ( $p$ 's $\leq .023)$, also if adjusted for IMT or plaque ( $p$ 's $\leq .025)$.

\subsubsection{Interaction negative affectivity and social inhibition}

Regarding the interaction between NA and SI results showed that higher interaction scores significantly related to higher WST-1 reduction scores $\left(\beta=.36, p=.022, R^{2}=.130\right.$; see Figure $\left.2 \mathrm{C}\right)$. This effect remained significant after controlling for BMI and $\operatorname{MAP}\left(\beta=.37, p=.032, \Delta R^{2}=.119\right)$. Additional controlling for IMT or presence of carotid plaques did not significantly change these 
results $(p$ 's $\leq .035)$. However, results became of borderline significance after controlling for BDI, CSSS, or both BDI and CSSS, in addition to BMI and MAP ( $p$ ' $s \leq .085)$, even if adjusted for IMT or presence of carotid plaques ( $p$ 's $\leq .090)$.

\section{Asssociations between WST-1 reduction scores, chronic stress and depressive symptom severity}

We further correlated WST-1 reduction scores with CSSS and BDI for the whole group in order to shed more light on the potential added value of Type D compared to depressive symptoms and chronic stress. Greater CSSS scores (WST-1 reduction score: $\beta=.30, p=.059, \Delta R^{2}=.090$ ), but not BDI scores (WST-1 reduction score: $\beta=.23, p=.147, \Delta R^{2}=.055$ ), were marginally associated with higher WST-1 reduction scores. Results remained similar after controlling for BMI, MAP, IMT, and presence of plaques (CSSS: WST-1 reduction score: $\beta=.30, p=.085, \Delta R^{2}=.083$; BDI: WST-1 reduction score: $\left.\beta=.23, p=.186, \Delta R^{2}=.050\right)$.

\section{DISCUSSION}

We investigated for the first time phagocytic NADPH oxidase-derived superoxide anion production of inflammatory M1 macrophages in CAD patients with and without Type D. As hypothesized, we found that CAD patients with Type D had higher WST-1 reduction scores of PMAactivated ex vivo isolated monocyte-derived M1 macrophages and thus higher macrophage superoxide anion production than patients without Type D. In addition, both Type D subscales 'negative affectivity' and 'social inhibition' and their interaction were associated with higher WST-1 reduction scores indicating higher superoxide anion production. Our results implicate that macrophages of CAD patients with Type $\mathrm{D}$ are characterized by an increased preparedness to release superoxide anions upon stimulation, i.e. in reaction to stimulating agents. The observed linear association with both Type D subscales suggests that this preparedness is higher with increasing negative affectivity and social inhibition, respectively.

Our findings are in line with previous studies reporting associations between Type D and immune measures (Conraads et al., 2006; Denollet et al., 2003; Einwik et al., 2011). In healthy participants, the presence of Type D was associated with a higher prevalence of cardiovascular risk 
factors including BMI, serum triglyceride levels, and higher levels of the inflammatory acute phase protein C-reactive protein (CRP; Einwik et al., 2011). Furthermore, Denollet and colleagues (2003) and Conraads and colleagues (2006) found higher plasma levels of the pro-inflammatory cytokine TNF- $\alpha$ and increased TNF- $\alpha$ soluble receptors in CHF patients with Type D compared to nonType D patients. Notably, higher TNF- $\alpha$ plasma levels relate to atherosclerosis development and progression (Libby et al., 2002; Kaptoge et al., 2014) and TNF- $\alpha$ soluble receptors may serve as a slow release reservoir for TNF- $\alpha$. Moreover, in CHF patients, Type D was associated with increased oxidative stress in terms of higher serum levels of the oxidant marker xanthine oxidase together with lower serum levels of the antioxidant marker Hsp70 (Kupper et al., 2009). However, this study did not assess ROS production to assess oxidative stress. Our study extends these findings by pointing to phagocytic NADPH oxidase-derived superoxide anion production by inflammatory M1 macrophages in CAD patients with Type D.

Both inflammatory M1 macrophages and superoxide anions play a pivotal role in the pathogenesis of atherosclerosis (Moore et al., 2013; Robbins et al., 2013). Increased production of NADPH-derived superoxide anions by non-phagocytic cells has been identified as a characteristic feature of CAD promoting atherosclerosis (Cai \& Harrison, 2000; Pennathur \& Heinecke, 2007; Szocs et al., 2002), supposedly by increasing endothelial dysfunction (Cai \& Harrison, 2000) and facilitating monocyte/macrophage infiltration into the intima (Guzik et al., 2006). Notably, increased endothelial dysfunction may result as a "response-to-injury" induced by superoxide anion release in reaction to stimulation by oxidized LDL present in atherosclerotic lesions (Libby, 2002; Stocker \& Keaney, 2004). Indeed, a very recent population-based study found Type D to be associated with endothelial dysfunction (van Dooren et al., 2016). In light of such reasoning, our findings may suggest a potential mechanism underlying the association between Type D and higher mortality in CAD patients.

We can only speculate about potential causes of the observed higher macrophage NADPHderived superoxide anion production with Type D in CAD patients. Inflammatory processes have been discussed as a potential biological basis of poor cardiac prognosis with Type D in CAD (Einwik et al., 2011; Kupper \& Denollet, 2007) and indeed, cardiac patients with Type D had higher 
basal inflammatory levels in terms of TNF- $\alpha$ (Conraads et al., 2006; Denollet et al., 2003). We speculate that inflammatory cytokines may prime macrophages to increased preparedness to superoxide anion release upon stimulation (Bedard \& Krause, 2007; Gauss et al., 2007). To understand the process leading to higher basal cytokine levels in cardiac patients with Type $\mathrm{D}$, it could be of interest to consider the two-stage model of vital exhaustion as proposed by Appels (1999). This model may also apply to Type D as a personality trait that likely predisposes to the experience of chronic stress. According to Appels (1999), overexertion or chronic stress leads to decreased immunocompetence (e.g. as a consequence of stress hormone secretion), thereby resulting in reactivation of systemic infections, the release of cytokines.

Clinical implications of our findings may relate to therapeutic modulation of Type D. Although Type D is known to be a personality trait, it is conceivable that an individual's level of distress could be modified by eligible interventions (Bagherian-Sararoudi et al., 2012). Two studies have shown that expanded cardiac rehabilitation programs reduced Type D scores of both subscales (Karlsson et al., 2007; Sogaro et al., 2010) and improved the quality of life among patients with Type D (Karlsson et al., 2007). Thus, future research on the effects of psychological interventions aimed at improving Type D and related phagocytic NADPH oxidase-derived superoxide anion production is warranted.

Strengths of our study are the careful patient recruitment including age-matching of the Type D patient groups and the use of the well-validated WST-1 assay. Moreover, we controlled for a variety of potential CAD-related confounders such as carotid IMT, presence of carotid plaques, BMI, and MAP, as well as the hitherto known macrophage superoxide anion release related psychological factors depressive symptom severity and chronic stress. The study also has its limitations. First, our study was cross-sectional and causality assumptions remain to be tested in prospective future investigations. Second, the present study focused on male CAD patients with and without Type D and may therefore not be generalizable to female patients with CAD or to patients with other diseases. Third, our experimental sample size requires confirmation in future studies with larger sample sizes. Fourth, the association of superoxide anion production as measured by the 
WST-1 assay with macrophage microbicidal activity in atherosclerotic lesions remains to be elucidated.

\section{CONCLUSION}

In sum, our data show an increased macrophage superoxide anion production in CAD patients with Type D compared to CAD patients without Type D. This suggests that macrophage superoxide anion production may play a mechanistic role in mediation of poor cardiac prognosis and higher morbidity and mortality in CAD patients with Type D. 
Acknowledgements: There are no conflicts of interest. We thank Jolina Lombardi, Halina Suter, Anja Lanz, Patricia Mueller, Marianne Kormann and Andrea Surtmann-Huguenin for their study assistance.

Funding sources: This work was funded by the Swiss National Science Foundation (Grant PP00P1_128565/1) (PHW). The funding source had no impact on study design, data collection, data analysis, manuscript writing, or the decision to submit the manuscript for publication. 


\section{REFERENCES}

Appels, A., 1999. Inflammation and the mental state before an acute coronary event. Ann Med. 78, 547-581.

Aukrust, P., Gullestad, L., Ueland, T., Damas, J.K., Yndestad, A., 2005. Inflammatory and antiinflammatory cytokines in chronic heart failure: Potential therapeutic implications. Ann Med. 37, $74-85$.

Babyak, M.A., 2004. What you see may not be what you get: a brief, nontechnical introduction to overfitting in regression-type models. Psychosom Med. 66, 411-421.

Bagherian-Sararoudi, R., Sanei, H., Attari, A., Afshar, H., 2012. Type D personality is associated with hyperlipidemia in patients with myocardial infarction. J Res Med Sci. 17, 543-547.

Barry-Lane, P.A., Patterson, C., van der Merwe, M., Hu, Z., Holland, S.M., Yeh, E.T., Runge, M.S., 2001. P47phox is required for atherosclerotic lesion progression in $\mathrm{ApoE}(-/-)$ mice. J Clin Invest. $108,1513-1522$.

Beck, A.T., Steer, R.A., Brown, G.K., 1996. Beck Depression Inventory - Second Edition. Manual. TX: The Psychological Corporation: San Antonio.

Bedard, K., Krause, K.H., 2007. The NOX family of ROS-generating NADPH oxidases: physiology and patophysiology. Physiol Rev. 87, 245-313.

Cai, H., Harrison, D.G., 2000. Endothelial dysfunktion in cardiovascular diseases. The role of oxidant stress. Circ Res. 87, 840-844.

Cathcart, M.K., 2004. Regulation of superoxide anion production by NADPH Oxidase in monocytes / macrophages: Contributions to atherosclerosis. Arterioscler Thromb Vasc Biol. 24, 23-28.

Chida, Y., Steptoe, A., 2009. The association of anger and hostility with future coronary heart disease: a meta-analytic review of prospective evidence. J Am Coll Cardiol. 53, 936-946.

Conraads, V.M., Denollet, J., Clerck, L.S., Stevens, W.J., Bridts, C., Vrints, C.J., 2006. Type D personality is associated with increased levels of tumour necrosis factor (TNF)- $\alpha$ and TNF- $\alpha$ receptors in chronic heart failure. Int J Cardiol. 113, 34-38.

Denollet, J., 2005. DS14: Standard Assessment of Negative Affectivity, Social Inhibition, and Typ D Personality, Psychosom Med. 67, 89-97.

Denollet, J., Conraads, V.M., Brutsaert, D.L., De Clerck, L.S., Stevens, W.J., Vrints, C.J., 2003. Cytokines and immune activation in systolic heart failure: the role of Type D personality. Brain Behav Immun. 17, 304-309.

Denollet, J., Pedersen, S.S., Vrints, C.J., Conraads, V.M., 2013. Predictive value of social inhibition and negative affectivity for cardiovascular events and mortalit in patients with coronary artery disease: The Type D personality construct. Psychosom Med. 75, 873-881.

Denollet, J., Sys, S.U., Stroobant, N., Rombouts, H., Gillebert, T.C., Brutsaert, D.L., 1996. Personality as independent predictor of long-term mortality in patients with coronary heart disease. Lancet, 347, 417-421. 
De Oliveira-Junior, E.B., Bustamante, J., Newburger, P.E., Condino-Neto, A., 2011. The human NADPH oxidase: primary and secondary defects impairing the respiratory burst function and the microbicidal ability of phagocytes. Scan J Immunol. 73, 420-427.

Dorshkind K., Montecino-Rodriguez E., Signer R.A., 2009. The ageing immune system: is it ever too old to become young again? Nat Rev Immunol. 9, 57-62.

Einwik, G., Dammen, T., Hrubos-Strom, H., Namtvedt, S.K., Randby, A., Kristiansen, H.A., Somers, V.K., Nordhus, I.H., Omland T., 2011. Prevalence of cardiovascular risk factors and concentration of C-reactive protein in Type D personality persons without cardiovascular disease. Eur $\mathbf{J}$ Cardiovasc Prev Rehabil. 18, 504-509.

Fulop T., Larbi, A., Douziech, N., Fortin C., Guérard, K.P., Lesur, O., Khalil, A., Dupuis, G., 2004. Signal transduction and functional changes in neutrophils with aging. Aging Cell, 3, 217-226.

Gauss, K.A., Nelson-Overton, L.K., Siemsen, D.W., Gao, Y., DeLeo, F.R., Quinn, M.T., 2007. Role of NF-kappaB in transcriptional regulation of the phagocyte NADPH oxidase by tumor necrosis factor-alpha. J Leukoc Biol. 82, 729-741.

Grande, G., Jordan, J., Kümmel, K., Struwe, C., Schubmann, R., Schulze, F., Unterberg, C., von Känel, R., Kudielka, B.M., Fischer, F., Herrmann-Lingen, C., 2004. Evaluation of the German Type D scale (DS14) and prevalence of the Type D personality pattern in cardiological and psychosomatic patients and healthy subjects. Psychother Psychosom Med Psychol. 54, 413-422.

Griendling, K.K., Sorescu, D., Ushio-Fukai, M., 2000. NAD(P)H Oxidase: Role in cardiovascular biology and disease. Circ Res. 86, 494-501.

Guzik, T.J., Sadowski, J., Guzik, B., Jopek, A., Kapelak, B., Przybylowski, P., Wierzbicki, K., Korbut, R., Harrison, D.G., Channon K.M., 2006. Coronary artery superoxide production and nox expression in human coronary artery disease. Arterioscler Thromb Vasc Biol. 26, 333-339.

Halliwell, B., 2006. Reactive Species and Antioxidants. Redox Biology is a Fundamental Theme of Aerobic Life. Plant Physiol. 141, 312-322.

Hautzinger, M., Keller, F., Kühner, Ch., 2006. Beck Depressions-Inventar Revision. Pearson: Frankfurt.

Kaptoge, S., Seshasai, S.R., Gao, P., Freitag, D.F., Butterworth, A.S., Borglykke, A., Di Angelantonio, E., Gudnason, V., Rumley, A., Lowe, G.D., Jorgensen, T., Danesh, J., 2014. Inflammatory cytokines and risk of coronary heart disease: new prospective study and updated meta-analysis. Eur Heart J. 35, 578-589.

Karlsson, M.R., Edström-Plüss, C., Held, C., Henriksson, P., Billing, E., Wallén, N.H., 2007. J Behav Med. 30, 253-261.

Kuebler, U., Ehlert, U., Zuccarella, C., Sakai, M., Stemmer, A., Wirtz, PH., 2013. An In Vitro Method to Investigate the Microbicidal Potential of Human Macrophages for Use in Psychosomatic Research. Psychosom Med. 75, 841-848.

Kupper, N., Denollet, J., 2007. Type D personality as a prognostic factor in heart disease. Assessment and mediating mechanisms. J Pers Assess. 89, 265-276.

Kupper, N., Gidron, Y., Winter, J., Denollet, J., 2009. Association between type D personality, depression, and oxidative stress in patients with heart failure. Psychosom Med. 71, 973-980. 
Libby, P., 2002. Inflammation in atherosclerosis. Nature, 420, 868-874.

Libby, P., Ridker, P.M., Hansson, G.K., 2011. Progress and challenges in translating the biology of atherosclerosis. Nature, 473, 317-325.

Libby, P., Ridker, P.M., Maseri, A., 2002. Inflammation and atherosclerosis. Circulation, 105, 11351343.

Lichtman, J.H., Bigger, J.T., Blumenthal, J.A., Frasure-Smith, N., Kaufmann, P.G., Lespérance, F., Mark, D.B., Sheps, D.S., Taylor, C.B., Froelicher, E.S., 2008. Depression and coronary heart disease: recommendations for screening, referral, and treatment: a science advisory from the American Heart Association Prevention Committee of the Council on Cardiovascular Nursing, Council on Clinical Cardiology, Council on Epidemiology and Prevention, and Interdisciplinary Council on Quality of Care and Outcomes Research: endorsed by the American Psychiatric Association. Circulation, 118, 1768-1775.

Lucas, A.D., Greaves, D.R., 2001. Atherosclerosis: role of chemokines and macrophages. Expert Rev in Mol Med. 3, 1-18.

Martinez, F.O., Sica, A., Mantovani, A. \& Locati, M., 2008. Macrophage activation and polarization. Front Biosci. 14, 453-461.

Moon, T.K., 1996. The Expectation-Maximization Algorithm. Signal Processing Magazine, 13, 47-60.

Moore, K.J., Sheedy, F.J., Fisher, E.A., 2013. Macrophages in atherosclerosis: a dynamic balance. Nat Rev Immunol. 13, 709-721.

Mosser, D.M., Edwards, P., 2008. Exploring the full spectrum of macrophage activation. Nat Rev Immunol. 8, 958-969.

Nathan, C., Shiloh, M.U., 2000. Reactive oxygen and nitrogen intermediates in the relationship between mammalian hosts and microbial pathogens. Proc Natl Acad Sci U S A. 97, 8841-8848.

Naqvi, T.Z., Lee, M.S., 2014. Carotid intima-media thickness and plaque in cardiovascular risk assessment. JACC Cardiovasc Imaging. 7, 1025-1038.

O’Dell, K.R., Masters, K.S., Spielmans, G.I., Maisto, S.A., 2011. Does Type D personality predict outcomes among patients with cardiovascular disease? A meta-analytic review. J Psychosom Med. $71,199-206$.

Pennathur, S., Heinecke, J.W., 2007. Oxidative stress and endothelial dysfunction in vascular disease. Curr Diab Rep. 7, 257-264.

Robbins, C.S., Hilgendorf, I., Weber, G.F., Theurl, I., Iwamoto, Y., Figueiredo, J.L., Gorbatov, R., Sukhova, G.K., Gerhardt, L.M.S., Smyth, D., Zavitz, C.C.J., Shikatani, E.A., Parsons, M., van Rooijen, N., Lin, H.Y., Husain, M., Libby, P., Nahrendorf, M., Weissleder, R., Swirski, F.K., 2013. Local proliferation dominates lesional macrophage accumulation in atherosclerosis. Nat Med. 19, 1166-1172.

Rosanski, A., 2014. Behavioral cardiology: currend advances and future directions. J Am Coll Cardiol. 64, 100-110.

Schulz, P., Schlotz, W., Becker, P., 2004. Trierer Inventar zum chronischen Stress. Hogrefe: Göttingen 
Shaw, A.C., Goldstein, D.R. \& Montgomery, R.R., 2013. Age-dependent dysregulation of innate immunity. Nat Rev Immunol. 13, 875-887.

Sogaro, E., Schinina, F., Burgisser, C., Orso, F., Pallante, R., Aloi, T., Vanni, D., Pazzagli, A., Fattirolli, F., 2010. Type D personality impairs quality of life, coping and short-term psychological outcome in patients attending an outpatient intensive program of cardiac rehabilitation. Monaldi Arch Chest Dis. 74, 181-191.

Stocker, R., Keaney, J.F., 2004. Role of oxidative modifications in atherosclerosis. Physiol Rev. 84, 1381-1478.

Szocs, K., Lassegue, B., Sorescu, D., Hilenski, L.L., Valppu, L., Couse, T.L., Wilcox, J.N., Quinn, M.T., Lambeth, J.D., Griendling, K.K., 2002. Upregulation of Nox-based NAD(P)H oxidases in restenosis after carotid injury. Arterioscler Thromb Biol. 22, 21-27.

Van Dooren, F.E., Verhey, F.R., Pouwer, F., Schalkwijk, C.G., Sep, S.J., Stehouwer, C.D., Henry, R.M., Dagnelie, P.C., Schaper, N.C., van der Kallen, C.J., Koster, A., Schram, M.T. \& Denollet, J. (2016). Association of Type D personality with increased vulnerability to depression: Is there a role for inflammation or endothelial dysfunction? - The Maastrich Study. Journal of Affective Disorders, 189, 118-125.

Watanabe, T., Yasunari, K., Nakamura, M., Maeda, K., 2006. Carotid artery intima-media thickness and reactive oxygen species formation by monocytes in hypertensive patients. J Hypertens. 20, 336-340.

Wirtz, P.H., Ehlert U., Emini, L., Suter, T., 2008. Higher body mass index (BMI) is associated with reduced glucocorticoid inhibition of inflammatory cytokine production following acute psychosocial stress in men. Psychoneuroendocrinology, 33, 1102-10.

Wirtz, P.H., von Kanel, R., Frey, K., Ehlert, U., Fischer, J.E., 2004. Glucocorticoid sensitivity of circulating monocytes in essential hypertension. Am J Hypertens. 17, 489-494.

Wykretowicz, A., Guzik, P., Kasinowski, R., Krauze, T., Bartkowiak, G., Dziarmaga, M., Wysocki, H., 2005. Augmentation index, pulse pressure amplification and superoxide anion production in patients with coronary artery disease. Int J Cardiol. 99, 289-294. 


\section{FIGURE LEGENDS}

\section{Legend to Figure 1}

WST-1 reduction scores in coronary artery disease (CAD) patients with Type D personality (Type D) and CAD patients without Type D (mean \pm SEM). CAD patients with Type D had higher WST-1 reduction scores than those without Type $\mathrm{D}(p<.001 * * *)$.

\section{Legend to Figure 2A}

The scatter plot shows a linear positive relationship between negative affectivity (NA) and WST-1 reduction scores $(p=.002)$.

\section{Legend to Figure 2B}

The scatter plot shows a linear positive relationship between social inhibition (SI) and WST-1 reduction scores $(p=.003)$.

\section{Legend to Figure 2C}

The scatter plot shows a linear positive relationship between the interaction of continuous negative affectivity and social inhibition (NAxSI) and WST-1 reduction scores ( $p=.022)$. 
Figure 1. Type D groups and WST-1 reduction scores

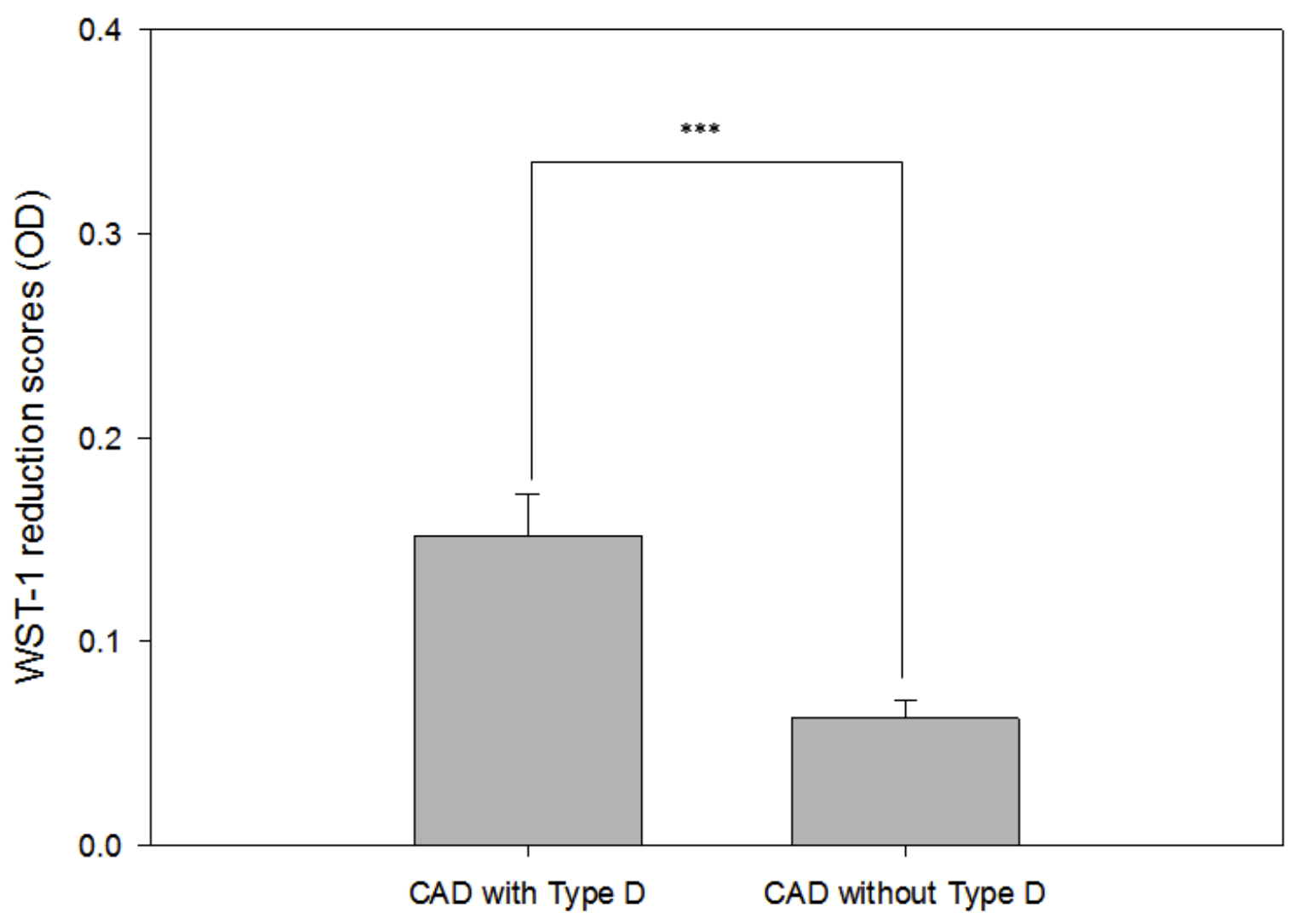


Figure 2A. Association between WST-1 reduction scores and the Type D subscale negative affectivity (NA)

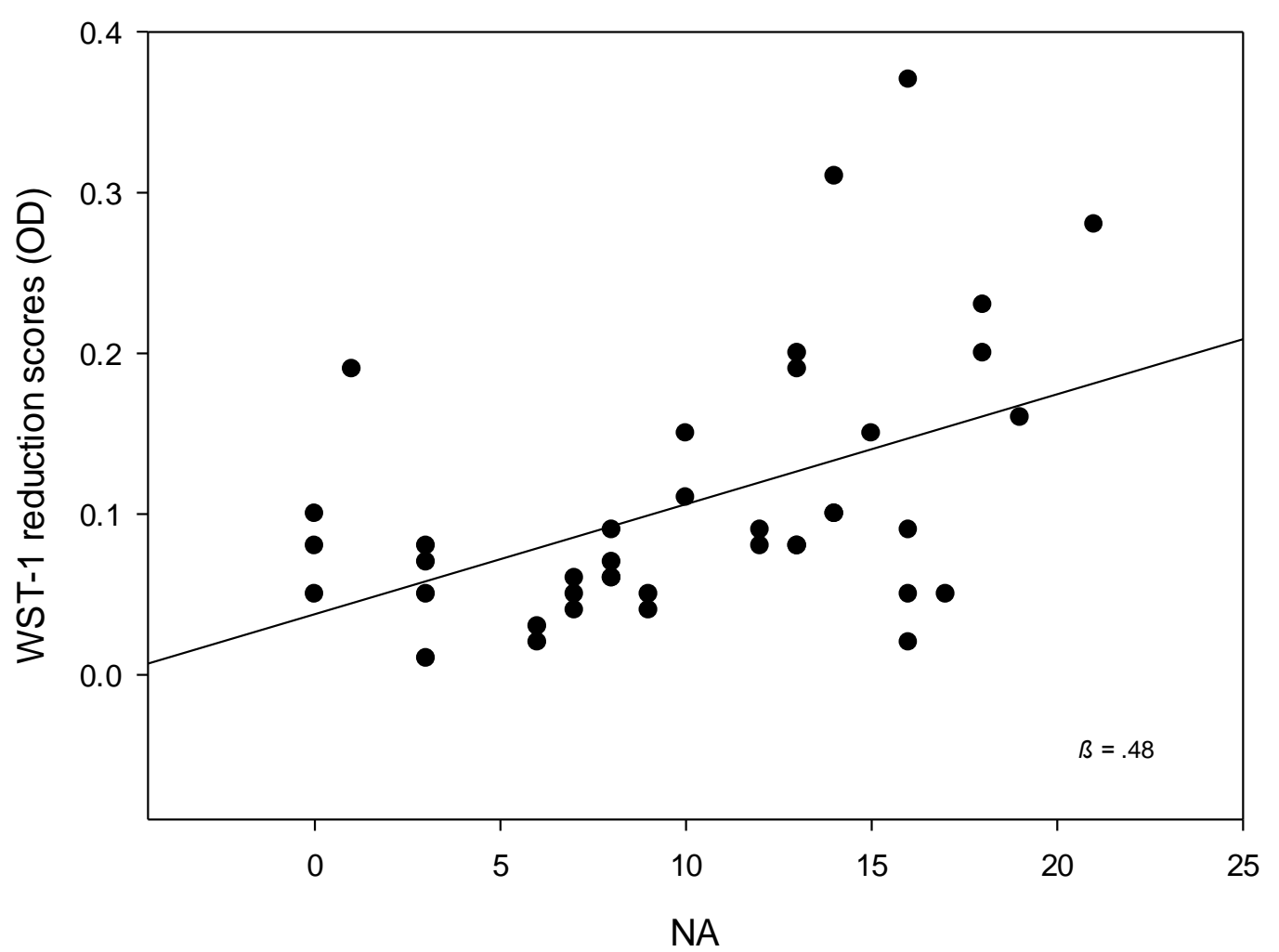


Figure 2B. Association between WST-1 reduction scores and the Type D subscale social inhibition (SI)

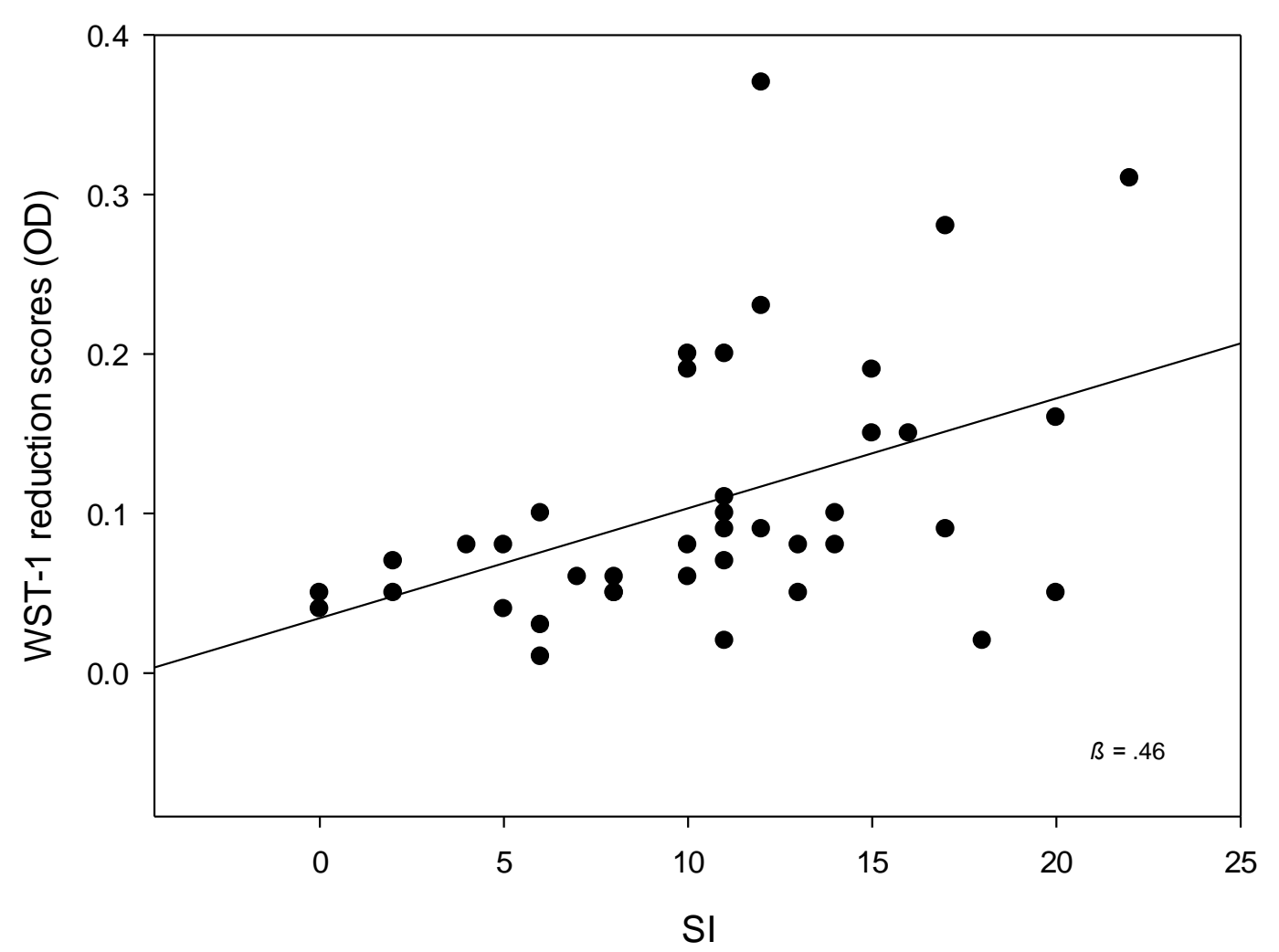


Figure 2C. Association between WST-1 reduction scores and the interaction of continuous negative affectivity and social inhibition (NAxSI)

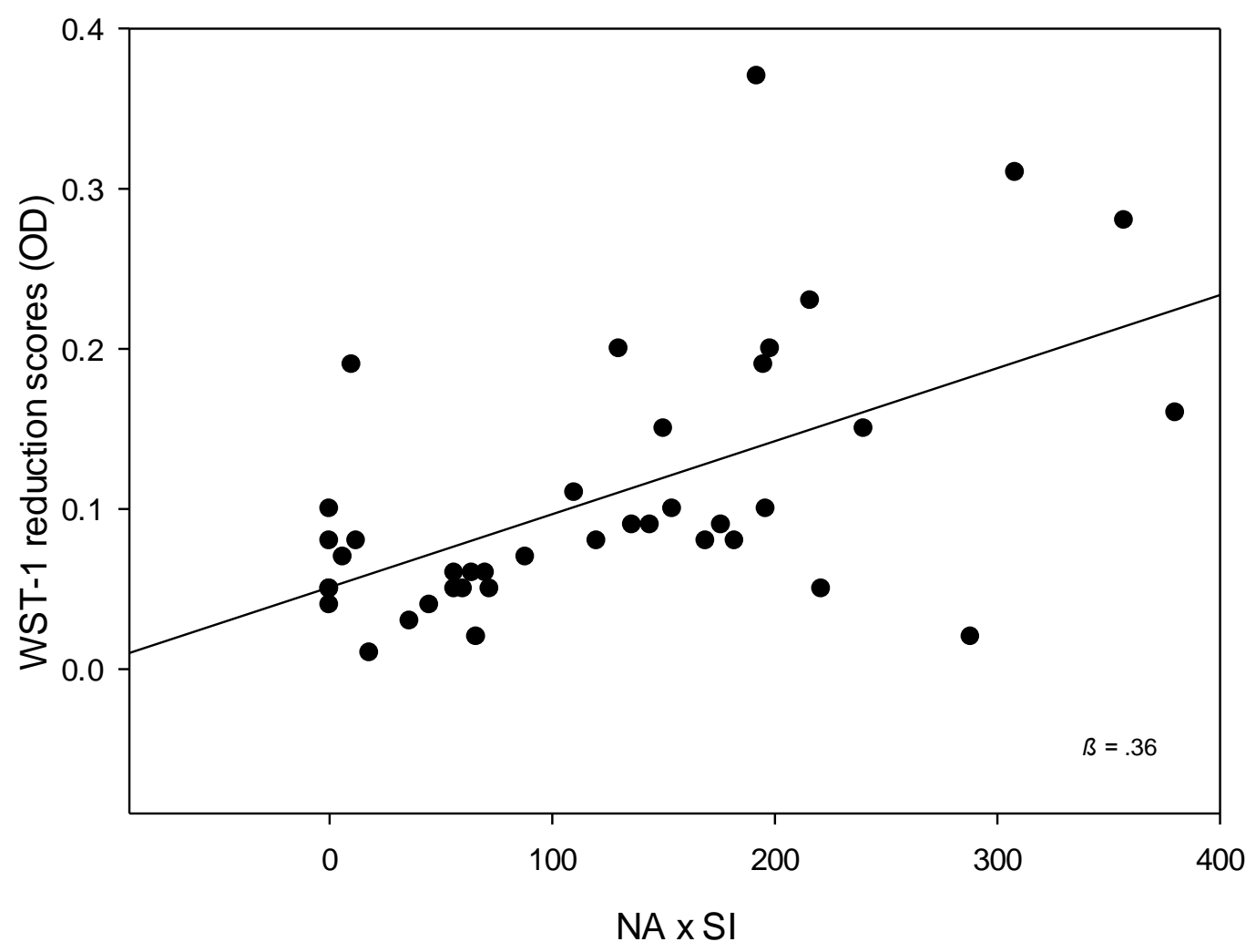


Table 1. Group characteristics of CAD patients with and without Type D personality

\begin{tabular}{|c|c|c|c|}
\hline & $\begin{array}{l}\text { CAD patients with } \\
\text { Type D } \\
\mathrm{n}=20\end{array}$ & $\begin{array}{l}\text { CAD patients without } \\
\text { Type D } \\
\mathrm{n}=20\end{array}$ & $P$-value \\
\hline Age (yr) & $66.7 \pm 2.2$ & $67.7 \pm 2.4$ & .75 \\
\hline $\operatorname{BMI}\left(\mathrm{kg} / \mathrm{m}^{2}\right)$ & $27.5 \pm 0.6$ & $27.8 \pm 0.5$ & .80 \\
\hline \multicolumn{4}{|l|}{ Blood pressure } \\
\hline $\mathrm{SBP}(\mathrm{mmHg})$ & $136.1 \pm 3.4$ & $137.7 \pm 4,14$ & .76 \\
\hline $\mathrm{DBP}(\mathrm{mmHg})$ & $74.8 \pm 2.0$ & $79.0 \pm 2.6$ & .21 \\
\hline MAP (mmHg) & $95.2 \pm 2.2$ & $98.6 \pm 3.0$ & .37 \\
\hline $\mathrm{LVEF} \leq 40 \%(\%)$ & $4(20)$ & $6(30)$ & .48 \\
\hline MI & $8(40)$ & $12(60)$ & .22 \\
\hline Number of diseased vessels & & & .20 \\
\hline $1(\%)$ & $5(25)$ & $10(50)$ & \\
\hline $2(\%)$ & $7(35)$ & $4(20)$ & \\
\hline $3(\%)$ & $8(40)$ & $6(30)$ & \\
\hline CABG (\%) & $5(25)$ & $6(30)$ & .73 \\
\hline IMT (\%) & $0.85 \pm 0.1$ & $0.87 \pm 0.2$ & .71 \\
\hline Plaques (\%) & $16(80)$ & $13(65)$ & .30 \\
\hline Current smokers (\%) & $2(10)$ & $1(5)$ & .56 \\
\hline \multicolumn{4}{|l|}{ Medication } \\
\hline Antiplatelet drugs (\%) & $19(95)$ & $17(85)$ & .30 \\
\hline Lipid-lowering drugs (\%) & $19(95)$ & $18(90)$ & .56 \\
\hline Beta-blockers (\%) & $12(60)$ & $14(70)$ & .52 \\
\hline ACE inhibitors (\%) & $6(30)$ & $8(40)$ & .52 \\
\hline AT2 antagonists (\%) & $7(35)$ & $4(20)$ & .30 \\
\hline Depressive symptom & $11.0 \pm 1.8$ & $5.2 \pm 1.0$ & .009 \\
\hline
\end{tabular}




\begin{tabular}{|l|c|c|c|}
\hline severity (BDI) & & & \\
\hline Chronic stress (CSSS) & $19.8 \pm 1.7$ & $9.4 \pm 1.5$ & $<.001$ \\
\hline
\end{tabular}

Values are means $\pm \mathrm{SEM}$; BDI $=$ Beck Depression Inventory; $\mathrm{BMI}=$ body mass index; $\mathrm{CABG}=$ coronary artery bypass graft; CAD = coronary artery disease; CSSS = chronic stress screening scale; DBP = diastolic blood pressure; IMT = intima-media thickness; LVEF = left ventricular ejection fraction; $\mathrm{MAP}=$ mean arterial blood pressure $; \mathrm{MI}=$ myocardial infarction; $\mathrm{SBP}=$ systolic blood pressure; $\mathrm{SEM}=$ standard error of mean; Type $\mathrm{D}=$ Type $\mathrm{D}$ personality 